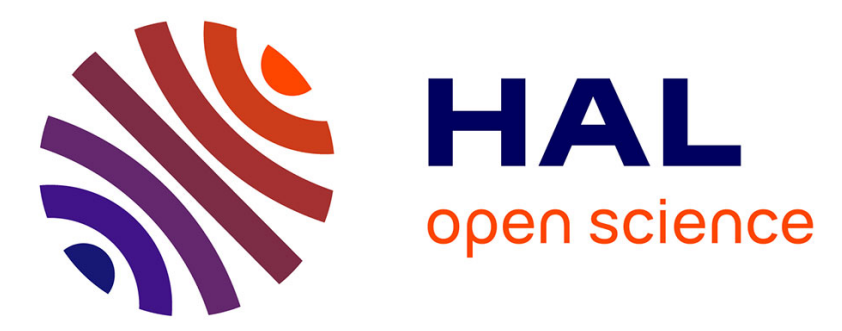

\title{
Aggregation behaviour of halato-telechelic polymers in non-polar solvents
}

\author{
A. Timbó, J. Higgins, D. Peiffer, C. Maus, P. Vanhoorne, R. Jérôme
}

\section{To cite this version:}

A. Timbó, J. Higgins, D. Peiffer, C. Maus, P. Vanhoorne, et al.. Aggregation behaviour of halatotelechelic polymers in non-polar solvents. Journal de Physique IV Proceedings, 1993, 03 (C8), pp.C871-C8-78. 10.1051/jp4:1993815 . jpa-00252246

\section{HAL Id: jpa-00252246 https://hal.science/jpa-00252246}

Submitted on 1 Jan 1993

HAL is a multi-disciplinary open access archive for the deposit and dissemination of scientific research documents, whether they are published or not. The documents may come from teaching and research institutions in France or abroad, or from public or private research centers.
L'archive ouverte pluridisciplinaire HAL, est destinée au dépôt et à la diffusion de documents scientifiques de niveau recherche, publiés ou non, émanant des établissements d'enseignement et de recherche français ou étrangers, des laboratoires publics ou privés. 


\title{
Aggregation behaviour of halato-telechelic polymers in non-polar solvents
}

\author{
A.M. TIMBÓ, J.S. HIGGINS, D.G. PEIFFER ${ }^{*}$, C. MAUS ${ }^{* *}$, P. VANHOORNE* and R. JÉRÔME** \\ Imperial College of Science, Technology and Medicine, London, U.K. \\ * Exxon Research and Engineering Company, Annandale, New Jersey, U.S.A. \\ ** University of Liège, Liège, Belgium
}

\section{INTRODUCTION}

Halato-telechelic polymers (HTP) are model ionomers consisting of a non-polar chain at each end of which one ionic group is attached ${ }^{1}$ (Figure 1). As a result of synthesis by living anionic polymerization and neutralization by metal acetates or alcoholates under well controlled conditions the properties of these polymers can be specified and controlled. The unusual behaviour of HTP in non-polar solvents results from the association of the end-groups which leads to the aggregation of the polymeric chains in the dilute regime and to the formation of a polymer network at concentrations higher than the critical concentration for gelation ${ }^{2,3}$. The interactions of the electric dipoles on the ionic groups are closely controlled by the molecular characteristics of the polymer backbone as well as parameters such as temperature, dielectric constant of the medium, and nature of the ionic group 4 . SANS and viscometry have proved to be powerful tools to follow the association processes which occurs between the ionomer molecules in solution ${ }^{5}$. In this paper we discuss the results of our SANS measurements on HTP, investigating the effects of changing the functional groups (carboxylate and sulphonate) and varying the molecular weight of the single chains. We compare the scattering results with the viscosity data on the same samples.

\section{EXPERIMENTAL}

\section{Sample preparation}

All the HTP samples used in this work, i.e., sodium carboxylated and sodium sulphonated polystyrene $\mathrm{PS}\left(\mathrm{CO}_{2} \mathrm{Na}\right)_{2}$ and $\mathrm{PS}\left(\mathrm{SO}_{3} \mathrm{Na}\right)_{2}$ - were prepared by anionic polymerization of pure and carefully dried styrene ${ }^{1-5,6}$. Living polystyryl dianions were initially obtained, whose deactivation with an excess of anhydrous carbon dioxide or propanesultone (depending on the ending group chosen) led to polymers in diacidic form. The molecular weights were obtained from gel permeation chromatography (GPC) in THF. The functionality was determined by potentiometric titration of the acidic groups with tetramethylamonium hydroxide in $9 / 1$ toluene/methanol (pure grade) mixtures. The polymeric salts were obtained by neutralizing the diacidic polymers. The neutralization was confirmed by infrared spectroscopy after distilling and vacuum drying the polymers.

\section{Preparation of the solutions}

HTP solutions for viscosity measurements were prepared by dissolving and successively diluting in toluene (spectrophotometric grade) an initial amount of the polymer, weighed using an analytical balance with an accuracy of $0.00002 \%$. The mixture of polymer and solvent was shaken for at least 48 hour, which is the minimum length of time required for equilibrium to be obtained. This minimum was determined by checking the reproducibility of viscosity measurements on the different telechelics used. Heating was shown to accelerate the process of dissolution, but was avoided in order to prevent modification in the aggregationdissociation history of the polymers. Due to the high viscosity of the solutions and to their tendency to shear thickening in capillaries, the solutions were not filtered but only centrifuged 24 hours before the measurements were performed. Although no visible impurities were found the bottom portion of the solutions were not used. The solutions for SANS experiments were prepared in a similar way, but using toluene- $d_{8},(99+$ atom $\%$ D). The solutions were analysed in Hellma quartz cells with $5 \mathrm{~mm}$ path. in order 
to maintain a reasonable transmission $(\sim 0.5)$ and to reduce the signal of incoherent scattering from the hydrogenous solvent.

\section{Instrumentation}

The rheological measurements on the HTP were carried out in modified Ubbelohde viscometers described by V. Desreux and J. Bischoff'. The small angle instrument LOQ, an accelerator-based neutron source (ISIS facility) located at Rutherford Appleton Laboratory (RAL) in the U.K., was used for all the SANS measurements. As described by Heenan and King ${ }^{8}$ the LOQ difractrometer uses neutron pulses containing wavelength in the range from 2.2 to $10 \AA$ at $25 \mathrm{~Hz}$. The pulses are recorded on a $64 \mathrm{~cm}$ diameter positionsensitive detector which is located at $4.3 \mathrm{~m}$ from the sample, with collimation apertures at $20 \mathrm{~mm}$ (after the supermirror bender) and 8-12 mm (sample diameter). This gives a $q$ range of $0.005-0.23 \AA^{-1}$ and provides information on sizes from 20 to $100 \AA$ in a single measurement.

\section{Analysis of the SANS data}

The data reduction and correction were executed according to procedure described by Heenan et al ${ }^{9}$ in the "Collete Users' Guide". Conventional Debye plots were made fitting the data obtained in order to determine molecular weights, $M$, and radius of gyration, $R_{g}$. For a polymer chain regarded as a Gaussian coil of $N$ segments, the mean square radius of gyration $\left\langle R_{g}{ }^{2}\right\rangle$ of these segments can be related to the coherent scattering, $S_{c o h}(q)$, through the expression

$$
S_{c o h}(q)=\frac{N^{2} 2}{\left(q^{2}\left\langle R_{g}{ }^{2}\right\rangle\right)}\left[\exp \left(-q\left\langle R_{g}{ }^{2}\right\rangle\right)-1+q^{2}\left\langle R_{g}{ }^{2}\right\rangle\right]
$$

The above equation applies to a single polymer molecule without intermolecular interactions. These are usually included via virial coefficients, $A_{2}, A_{3}, \ldots$ At small values of $q R_{g}$, Equation 1 reduces to a very simple form, so that for $R_{g} \ll 1$ and including intermolecular interactions, the scattered intensity can be expressed as Equation 2 follows here

$$
\frac{K^{*} c}{I(q)}=M^{-1}\left(1+\frac{q^{2}\left\langle R_{g}{ }^{2}\right\rangle}{3}\right)+2 A_{2} c+3 A_{3} c^{2}+\ldots \ldots
$$

where $K^{*}=\left(F K N_{A}\right) / m^{2}$ and $F$ contains geometrical parameters of the instrument, $K$ is the contrast factor, $N_{A}$ is the Avogadro's number and $m$ is the segment molecular weight. Figure 2 shows the Debye fit for one these solutions used. The Debye function was deliberated chosen to make comparison with ionomers and to allow a large $q$-range to be used. Surprisingly it was observed to fit the shape of the scattering from the HTP solutions equally well within the limitations of the instrument. In this plot, as in all the others, extra scattering can be found at low $q$ values, above the Debye fit limit, whose origin is difficult to identify. It could be attributed either to large impurities in the solution, which is unlikely given that they were centrifuged, or to residue arising from the cell subtraction. This problem does not strongly affect the results since the range of fitting chosen excluded those scattering signals in all cases. Further treatment of data (calculations and plotting) was accomplished using Microsoft Excel 4.0 and CricketGraph 3.1.

\section{RESULTS AND DISCUSSION}

\section{Scattering}

Results from solutions of sodium sulphonate and sodium carboxylate polystyrene $-\mathrm{PS}\left(\mathrm{SO}_{3} \mathrm{Na}\right)_{2}$ and $\mathrm{PS}\left(\mathrm{CO}_{2} \mathrm{Na}\right)_{2}$ - in toluene were used to evaluate the strength of the interaction of the two different ionic groups and their influence on the extent of aggregation between these polymers. The effect of the initial molecular weight ( $M_{w}$ of a single chain) on the aggregation process was also studied for both kinds of ionic groups. Figures 3 to 6 show the behaviour of the apparent molecular weight $\left(M_{w, \text { app }}\right)$ and radius of gyration 
$\left(R_{g, a p p}\right)$, as a function of concentration, for $\mathrm{PS}\left(\mathrm{SO}_{3} \mathrm{Na}\right)_{2}$ with 9000 and $18000 \mathrm{~g} / \mathrm{mol} M_{w}$, and for $\mathrm{PS}\left(\mathrm{CO}_{2} \mathrm{Na}\right)_{2}$ with 8000 and $20000 \mathrm{~g} / \mathrm{mol} M_{w}$. A clear difference in the shape of the curves can be seen between the polymers with lower $M_{w}$ and the ones with higher $M_{w}$ for both sulphonated and carboxylated HTP. This suggests two different processes of association. Previous studies on ionomers ${ }^{10}$ have revealed that the polymer with $105300 \mathrm{~g} / \mathrm{mol} M_{w}$ and $1.38 \mathrm{~mol} \%$ sulphonation level associated via a mechanism which could be described by the open association model, characterized by a concentration dependent continuous distribution of aggregate molecular weights. An ionomer with $105300 \mathrm{~g} / \mathrm{mol} M_{w}$ and $0.95 \mathrm{~mol} \%$ sulphonation level, which we are currently investigating, has been shown to form aggregates whose molecular weights were more in agreement with some form of the closed association model. In this type of behaviour aggregates of a narrow molecular weight range (independent of concentration) dominate down to very low concentrations at which point single molecules appear. The single chain lengths of the telechelics with lower $M_{w}$ are comparable to the average length between ions of the $1.38 \mathrm{~mol} \%$ ionomer, while the single chains lengths of the telechelics with higher $M_{w}$ are comparable to the average length between ions of the $0.95 \mathrm{~mol} \%$ ionomer. Although the extrapolated values of the $M_{w, a p p}$ and $R_{g, a p p}$ at zero concentration do not correspond to the values expected for a single chain either in the telechelics of lower $M_{w}$ or in those of higher $M_{w}$, it appears, from the shape of the curves in Figures 3-6, that:

1. The dependence of the $M_{w, a p p}$ and the $R_{g, a p p}$ for $\mathrm{PS}\left(\mathrm{SO}_{3} \mathrm{Na}\right)_{2}$ with $9000 \mathrm{~g} / \mathrm{mol} M_{w}$ and for $\mathrm{PS}\left(\mathrm{CO}_{2} \mathrm{Na}\right)_{2}$ with $8000 \mathrm{~g} / \mathrm{mol} M_{w}$ is governed by an open association process;

2. For $\mathrm{PS}\left(\mathrm{SO}_{3} \mathrm{Na}\right)_{2}$ with $18000 \mathrm{~g} / \mathrm{mol} M_{w}$ and for $\mathrm{PS}\left(\mathrm{CO}_{2} \mathrm{Na}\right)_{2}$ with $20000 \mathrm{~g} / \mathrm{mol} M_{w}$, such dependence is driven by a closed association process.

For both lower and higher $M_{w}$, it can be observed (Figures 3 and 5) that the carboxylated telechelics form aggregates with apparent molecular weights larger than those formed by the sulphonated polymers. The explanation for this fact could be found in the effect that the steric hindrance of the ionic groups have on the aggregate dimensions. The increase in the size of the $\mathrm{NaSO}_{3}$ - group as compared to the $\mathrm{NaCO}_{2}$ - hinders the ability of the chains to optimally interact. This steric hindrance effect influences the apparent radius of gyration of the aggregates, but its magnitude is dependent on the molecular weight of the single chains. At low molecular weights the $R_{g, a p p}$ is only marginally larger for the sulphonate-associating group than for the carboxylate-containing polymers (Figure 4). As the $M_{w}$ of a single chain is doubled (Figure 6), we observe an increase in the aggregate size overall as anticipated, but the increase is most pronounced in the carboxylate-containing polymer.

\section{Comparison with viscosity}

As with the SANS results, the rheological data (Figure 7 and 8) probes the influence of $M_{w}$ and the type of ionic group on the aggregation process. A difference is, however, observed between the results obtained through these two techniques. From these rheological results and comparison with published viscosity data for PS ${ }^{11}$ (Figure 9) it could appear, at a first glance, that it would be possible to observe individual chains at low concentration, which are apparently not detectable by SANS ( $c f$. Figures 3 and 7 , for example). Detecting single chains from viscometry could be attributed to aggregate dissociation occurring as a consequence of the high shear rate to which the polymer is submitted in the viscometer capillary, particularly in the case of solutions of structured polymers sensitive to any external perturbation. In the SANS measurements described here, however, the solutions were examined under quiescent conditions, and it would be possible that this could give rise to the observed difference in the results from these techniques. From another point of view, however, the explanation for such difference seems to be more reasonably found in the relationship viscosity-volume. The frictional properties of polymer molecules in solution show that the intrinsic viscosity is proportional to the effective hydrodynamic volume of the molecule in solution divided by its molecular weight. The effective volume is proportional to the cube of a linear dimension of the randomly coiling chain. If the root-mean-square distance between the ends of the chain, $\left\langle S^{2}\right\rangle^{1 / 2}$, is taken, then 


$$
[\eta]=\frac{F\left(S^{2}\right)^{3 / 2}}{M}
$$

where $\Phi$ is a universal constant for all flexibly coiled polymers. Equation 3 is consistent with the data obtained for the radii of gyration. As a proportionality factor between the radius of gyration and the hydrodynamic volume, $\Phi$ is only related to the expansion of the coil in the relevant solvent. The small values observed for the viscosities of the more dilute solutions should not represent individual chains but perhaps some sort of primary aggregation unit whose $R_{g, a p p}$ does not differ too much from those of the single chains ( $c f$. Figures 4 and 6). A salient feature of these HTP pertains to the influence of aggregation on the rheological characteristics of the solutions. As the polymer concentration is increased ( $c f$. the SANS results in Figure 5), the aggregation molecular weight is also enhanced. These results apply to both sulphonate and carboxylate-containing polymers, although differences are found in these two materials. This is clearly an effect of the strength of the ionic interactions. In addition, up to a specific concentration, the sulphonate-containing aggregates grow at a more rapid rate than the carboxylate-containing aggregates. This is observed in the marked change in slope of the $\eta_{\text {red }}$-concentration profiles. Both polymers at concentrations lower than about $2.0 \mathrm{~g} / \mathrm{dl}$ have lower viscosity, but at higher concentration a rapid rise in viscosity is observed. This is undoubtedly due to the formation of a three-dimensional network held together by ionic associations. Since these associations are labile, the network does reversibly break up upon dilution.

\section{CONCLUSIONS}

Although the unusual behaviour of $M_{w, a p p}$ and $R_{g, a p p}$ exhibited by the telechelics investigated in this study does not allow a completely clear understanding of the mechanism(s) governing the association of their molecules, it gives the possibility to develop a probable hypothesis. The concentration dependent distribution of aggregate molecular weights occurring for the lower $M_{w}$ HTP is continuous which indicates an open association. The results for the higher $M_{w}$ HTP suggest a closed association. However the apparent absence of single chains in the quiescent dilute solutions indicates that these models can not be fitted exactly. Double association processes, closely related to the two processes described above, such as aggregation of aggregates, could be occurring. A mathematical treatment would have to be developed with the aid of further neutron scattering, rheology and light scattering experiments. The schematic pictures in Figure 10 represent possible structures for the association of these HTP in a non-polar solvent based on the SANS and rheological measurements. Besides providing significant information on the molecular weight and size distribution of the aggregates, the results of the investigation on HTP also allowed us to confirm our expectations concerning aggregation in ionomers (NaSPS). The prediction suggested by data from ionomers with random placement of the ionic groups that ions must be attached to or near the ends of the chains for aggregation to occur, when the $M_{w}$ of the single chain is lower than a critical value ${ }^{12}$, is confirmed by the fact that association occurs for the telechelics, which have even lower and only two salt groups per chain.

\section{REFERENCES}

1. Broze, G., Jèrôme, R. and Teyssié, Ph; Macromolecules, 15, 920 (1982).

2. Broze, G., Jèrôme, R. and Teyssié, $\mathrm{Ph}$; Macromolecules, 14, 224 (1981).

3. Broze, G., Jèrôme, R., and Teyssié, Ph; Macromolecules, 15, 1300 (1982).

4. Broze, G., Jèrôme, R., Teyssié, Ph and Gallot; R., J. Polymer Sci., Polym. Lett. Ed., 19, 415 (1981).

5. Lantman, C. W., Mcknight, W. J., Higgins, J. S., Peiffer, D. G., Sinha, S. K. and Lundberg, 
R. D.; Macromolecules, 21, 1339 (1988).

6. Vanhoorne, P. and Jérôme, R., unpublished

7. Desreux, V. and Bischoff, J., Bull. Soc. Chim. Belg., 59, 93 (1950).

8. Heenan, R. K. and King, S. M., J. Non-Cryst. Sol., North-Holland, 150, 153 (1992).

9. Heenan, R. K., King, S. M., Osborn, R. and Stanley, H. B., "Collete Users' Guide", Rutherford Appleton Lab. Report RAL-89-128 (1989).

10. Pedley, A. M.; PhD Thesis - Imperial College, London (1990).

11. Kniewkse and W-M. Kulicke; Makrom. Chem., 184, 2173 (1983).

12 Timbó, A., PhD Thesis - Imperial College, London (1993). 


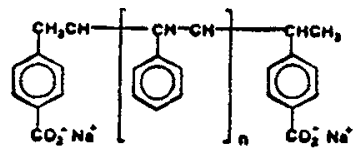

Figure 1. Sodium carboxylate polystyrene telechelic.

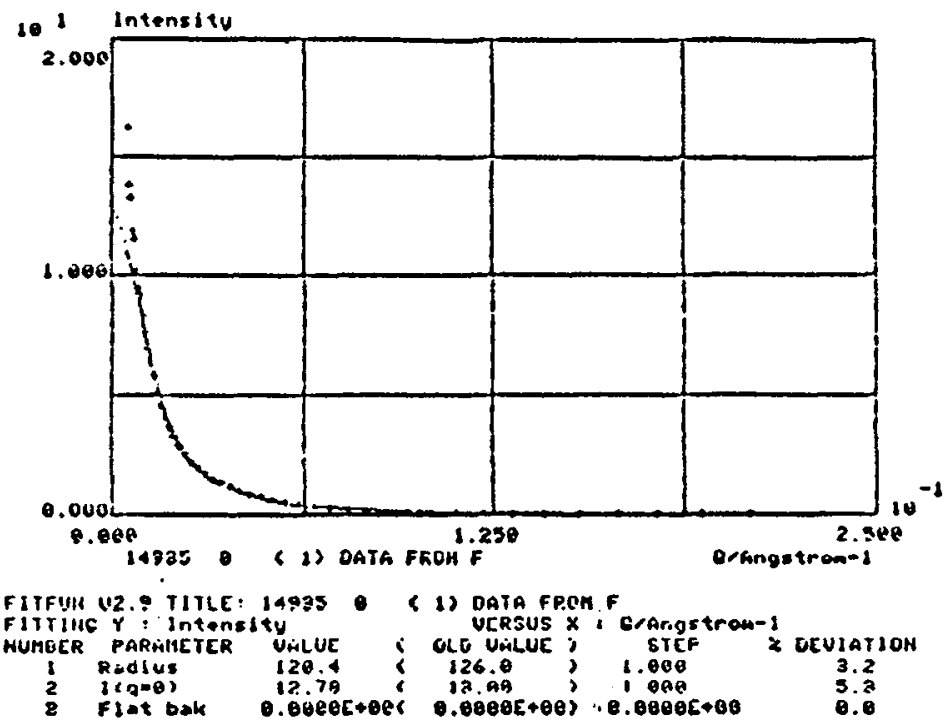

Figure 2. Debye fit for a $2.5 \mathrm{~g} /$ dl solution of $\mathrm{PS}\left(\mathrm{SO}_{3} \mathrm{Na}\right)_{2}\left(18000 \mathrm{~g} / \mathrm{mol} \mathrm{M}_{w}\right)$ in toluene.

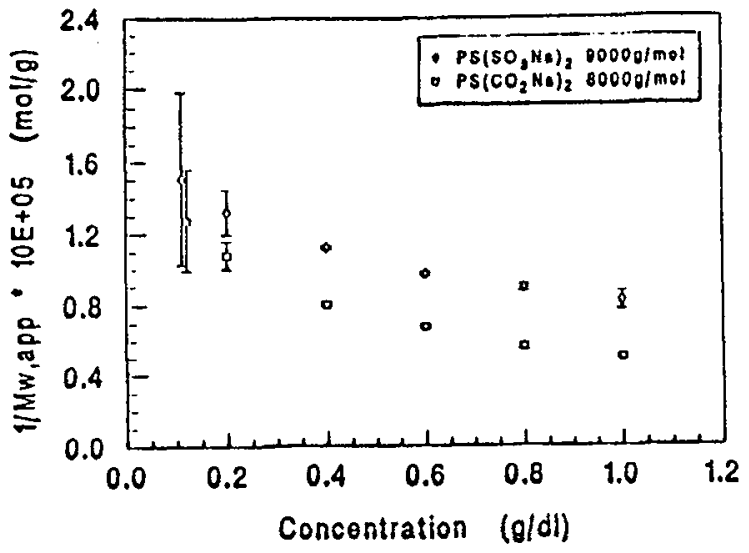

Figure 3. Dependence of the aggregates $M_{w, a p p}$ on the concentration of the solutions - SANS data for the lower single chain molecular weights. 


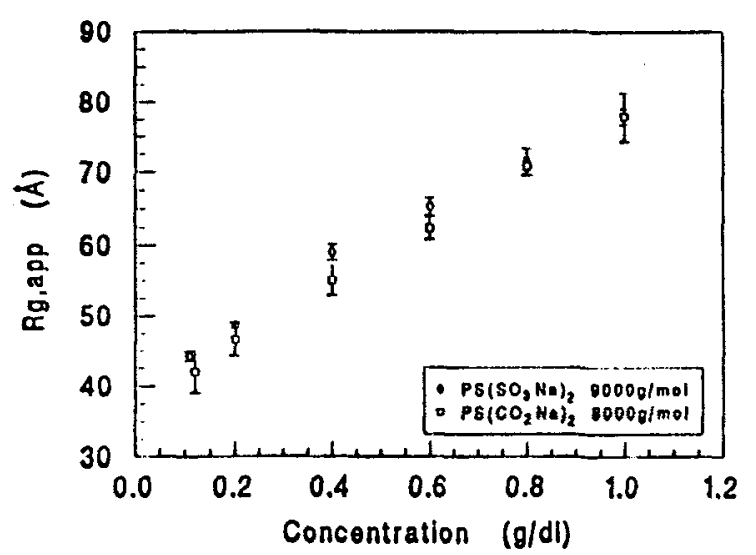

Figure 4. Dependence of the aggregates $R_{8, a p p}$ on the concentration of the solutions - SANS data for the lower single chain molecular weights.

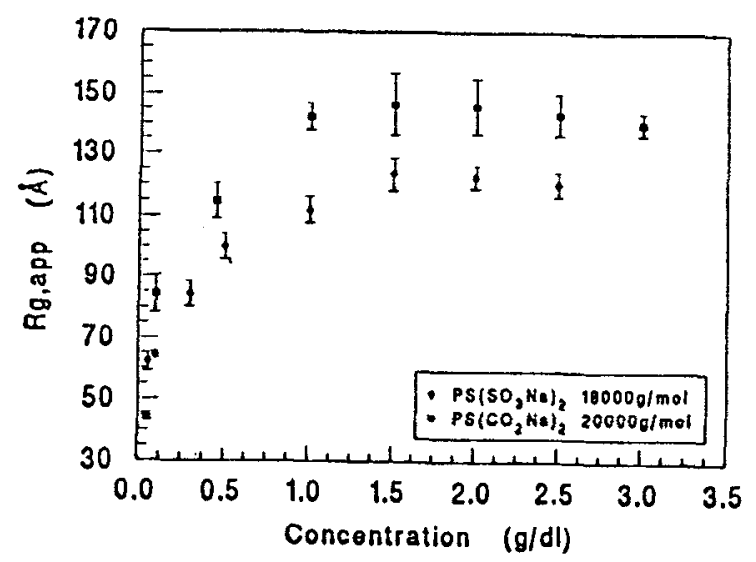

Figure 6. Dependence of the aggregates $R_{g, a p p}$ on the concentration of the solutions - SANS data for the higher single chain molecular weights.

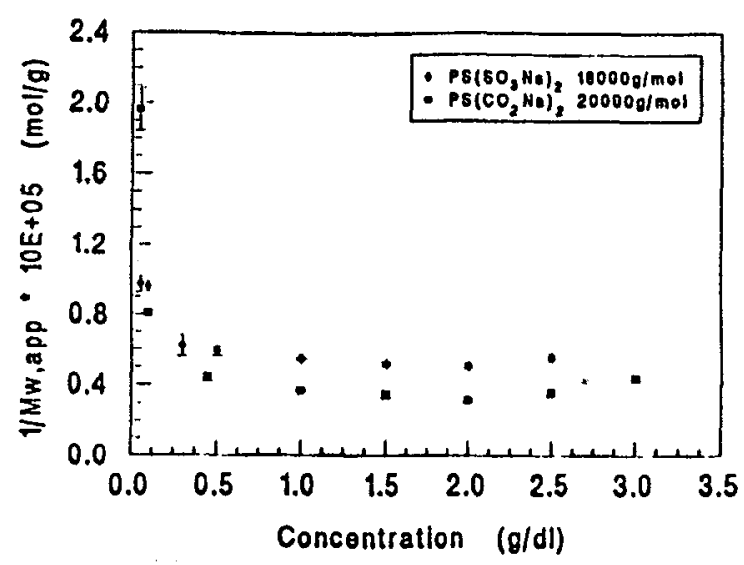

Figure 5. Dependence of the aggregates $M_{w, a p p}$ on the concentration of the solutions - SANS data for the higher single chain molecular weights.

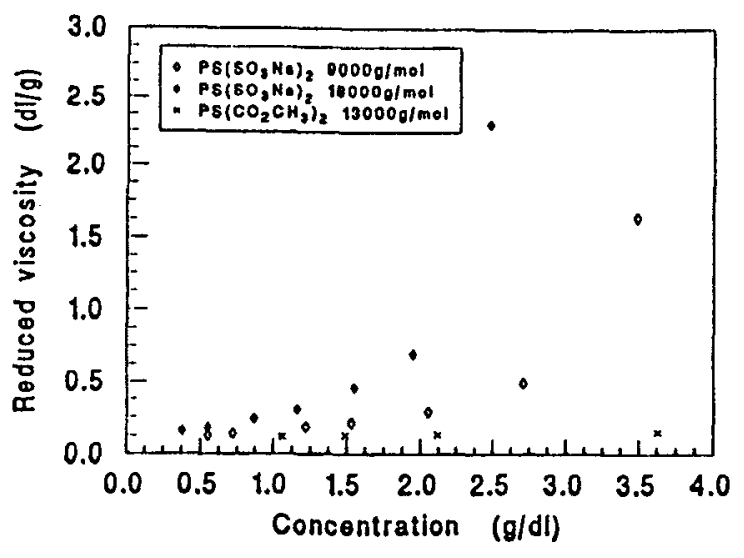

Figure 7. Effect of the concentration and single chain molecular weight on the reduced viscosity of sodium sulphonated telechelics in toluene at $25^{\circ} \mathrm{C}$; Comparison with an esther end group sample. 


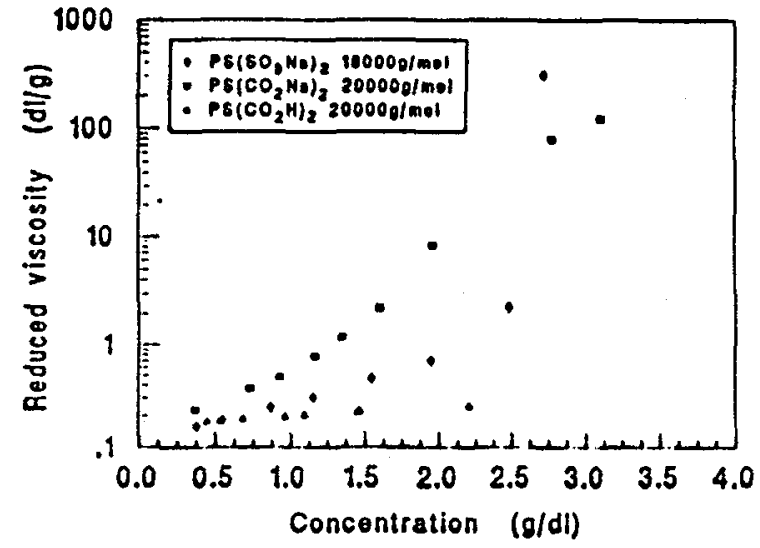

Figure 8. Effect of the concentration and type of ionic group on the reduced viscosity of sodium sulphonated telechelics in toluene at $25^{\circ} \mathrm{C}$;

Comparison with a non-neutralized (acidic end group) sample.

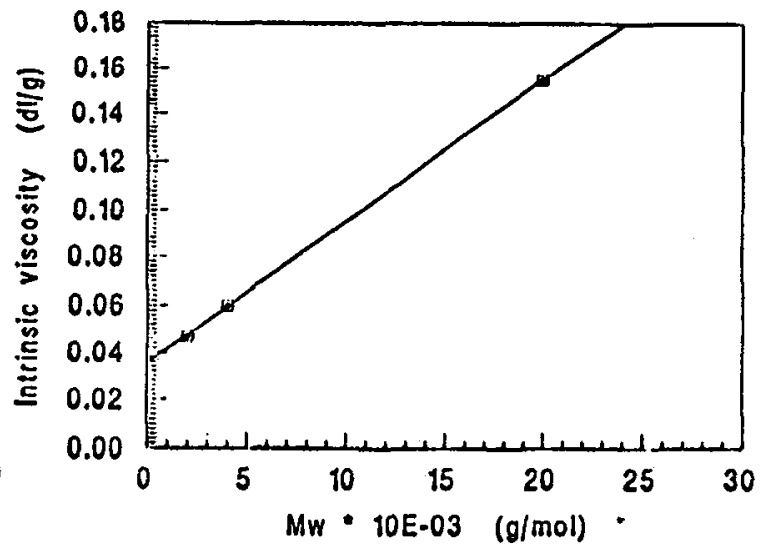

Figure 9. Influence of the molecular weight on she intrinsic viscosity (lim $c \rightarrow 01$ red $)$ [1]], of Polystyrene in toluene at

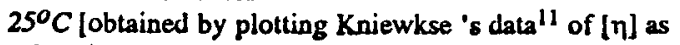
a function of $M_{w}$ ].
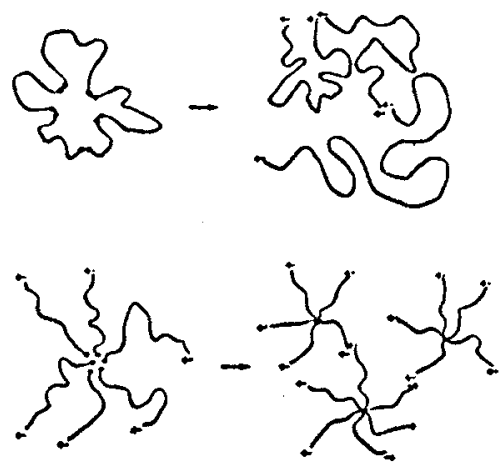

Figure 10. Possible structures of the HTP aggregates in non-polar solven 